\title{
Vorinostat-eluting poly(DL-lactide-co-glycolide) nanofiber-coated stent for inhibition of cholangiocarcinoma cells
}

This article was published in the following Dove Press journal:

International Journal of Nanomedicine

17 October 2017

Number of times this article has been viewed

\author{
Tae Won Kwak ${ }^{1, *}$ \\ Hye Lim Lee ${ }^{2, *}$ \\ Yeon Hui Song ${ }^{2}$ \\ Chan $\mathrm{Kim}^{3}$ \\ Jungsoo Kim² \\ Sol-ji Seo ${ }^{2}$ \\ Young-II Jeong ${ }^{2}$ \\ Dae Hwan Kang ${ }^{2,4}$
}

'Medical Convergence Textile Center, Gyeongbuk, Republic of Korea;

${ }^{2}$ Biomedical Research Institute, Pusan National University Hospital, Pusan, Republic of Korea; ${ }^{3}$ Amogreentech Co. Ltd. Gyeonggi-do, Republic of Korea;

${ }^{4}$ Research Institute for Convergence

of Biomedical Science and Technology, Pusan National University Yangsan

Hospital, Gyeongnam, Republic

of Korea

*These authors contributed equally to this work

Correspondence: Young-II Jeong Biomedical Research Institute, Pusan National University Hospital, 179 Gudeok-ro, Seo-gu, Busan 4924I, Republic of Korea

Tel +82 553603873

Fax +82553603879

Email nanomed@naver.com

Dae Hwan Kang

Research Institute for Convergence of Biomedical Science and Technology,

Pusan National University Yangsan

Hospital, 20, Geumo-ro, Mulgeum-eup,

Yangsan, Gyeongnam 506I2,

Republic of Korea

Tel +82 553603870

Fax +82 553603879

Email sulsulpul@naver.com
Purpose: The aim of this study was to fabricate a vorinostat (Zolinza $\left.{ }^{\mathrm{TM}}\right)$-eluting nanofiber membrane-coated gastrointestinal (GI) stent and to study its antitumor activity against cholangiocarcinoma (CCA) cells in vitro and in vivo.

Methods: Vorinostat and poly(DL-lactide-co-glycolide) dissolved in an organic solvent was sprayed onto a GI stent to make a nanofiber-coated stent using an electro-spinning machine. Intact vorinostat and vorinostat released from nanofibers was used to assess anticancer activity in vitro against various CCA cells. The antitumor activity of the vorinostat-eluting nanofiber membrane-coated stent was evaluated using HuCC-T1 bearing mice.

Results: A vorinostat-incorporated polymer nanofiber membrane was formed on the surface of the GI stent. Vorinostat was continuously released from the nanofiber membrane over 10 days, and its release rate was higher in cell culture media than in phosphate-buffered saline. Released vorinostat showed similar anticancer activity against various CCA cells in vitro compared to that of vorinostat. Like vorinostat, vorinostat released from nanofibers induced acetylation of histone H4 and inhibited histone deacetylases $1 \cdot 3 \cdot 4 / 5 / 7$ expression in vitro and in vivo. Furthermore, vorinostat nanofibers showed a higher tumor growth inhibition rate in HuCC-T1 bearing mice than vorinostat injections.

Conclusion: Vorinostat-eluting nanofiber membranes showed significant antitumor activity against CCA cells in vitro and in vivo. We suggest the vorinostat nanofiber-coated stent may be a promising candidate for CCA treatment.

Keywords: vorinostat, nanofiber, GI stent, cholangiocarcinoma, drug eluting

\section{Introduction}

Metal stents are used to maintain drainage when the gastrointestinal (GI) duct is blocked by tumor growth or inflammation. ${ }^{1-4}$ Cholangiocarcinoma (CCA), which is derived from the epithelium of the bile ducts, is frequently diagnosed at an advanced state and typically has a poor prognosis with a short median survival time..$^{5-7}$ Palliative endoscopic stenting for advanced CCA is frequently conducted to prolong patient survival when curative options, such as surgical removal, is not possible. ${ }^{1,4,5}$ Covered or uncovered metal stents only provide mechanical palliation of the obstructed bile duct with absence of anticancer activity when biliary obstruction has occurred due to tumor growth. ${ }^{8,9}$ Drug-eluting stents (DESs) have been extensively investigated for the treatment of GI cancer, including CCA. ${ }^{10-14}$ Paclitaxel-eluting stents are known to have anti-tumor efficacy in rabbit esophageal squamous carcinoma models, ie, paclitaxel-eluting stents reduced the tumor area to a third of the metal stent. ${ }^{11}$ Kim et al also reported that paclitaxel-eluting stents inhibited CT26 colon tumor growth in a 
mouse tumor xenograft model. ${ }^{12}$ In this report, they showed that the volume of tumors treated with paclitaxel-eluting membranes were more than 50\% smaller than those treated with paclitaxel injection. Previously, we also reported that a sorafenib-eluting GI stent had anti-tumor efficacy against HuCC-T1 CCA cells in vitro and in animal tumor xenograft models. ${ }^{13}$ Anticancer agent-eluting stents for CCA may represent a prospective treatment option to prolong patient survival.

Suberoylanilide hydroxamic acid (Zolinza ${ }^{\mathrm{TM}}$ [vorinostat]), a zinc ion chelator that acts on the active site of histone deacetylases (HDACs), is a common HDAC inhibitor and regulates transcription through acetylation/deacetylation processes. ${ }^{14-16}$ Vorinostat is the first HDAC inhibitor approved by the US Food and Drug Administration for the treatment of cutaneous $\mathrm{T}$ cell lymphoma. ${ }^{17}$ Recent clinical trials demonstrated that vorinostat is a safe and effective treatment for GI cancer. ${ }^{18}$ Vorinostat has been reported to have inhibitory effects against various CCA cells in vitro and in vivo alone and in combination with other drugs. ${ }^{19-22}$

Since nanofiber mats have a similar scale to biological interface and superior biocompatibility, they have been extensively investigated in the biomedical field. ${ }^{23-27}$ Due to these intrinsic properties, nanofibers have been applied in scaffold materials, biomedical devices, and drug delivery devices. ${ }^{24-27}$ Nanofibers have been explored as a drug delivery device for bioactive molecules because they have large surface areas and the small fiber thickness and drug release behavior can be easily controlled. ${ }^{26,27}$ These unique features of nanofibers make them a suitable device for drug-eluting membranecovered GI stents. ${ }^{12}$

In this study, we fabricated a nanofiber-coated GI stent for vorinostat-eluting stent development. Vorinostat-incorporated nanofibers were fabricated using an electro-spinning technique and simultaneously coated onto a GI stent. Their physicochemical properties, such as morphology and drug release behavior, were investigated. Furthermore, their anticancer activities against CCA cells were studied in vitro and in a tumor xenograft model.

\section{Materials and methods Materials}

Poly(DL-lactide-co-glycolide) (PLGA 503H) was purchased from Evonik Co. Inc. (Darmstadt, Germany). Vorinostat was purchased from LC Laboratories ${ }^{\circledR}$ Co. (Woburn, MA, USA). Tetrahydrofuran (THF), acetone, and dimethyl sulfoxide (DMSO) was purchased from Sigma-Aldrich Co. (St Louis, MO, USA). Silicon-membrane-covered stent for bile duct was purchased from M.I. Tech. (Pyeongtaek-si, Korea). Cell culture supplies such as RPMI 1640 media and fetal bovine serum (FBS) were purchased from ThermoFisher Scientific (Waltham, MA, USA). All reagent and organic solvents used were HPLC-grade.

\section{Fabrication of vorinostat-incorporated nanofiber membrane and coating of the Gl stent}

Vorinostat-incorporated nanofiber was coated onto a GI stent as shown in Figure 1. Vorinostat $(50 \mathrm{mg})$ was dissolved in $0.5 \mathrm{~mL} \mathrm{THF} / 9.5 \mathrm{~mL}$ acetone solution. To this solution, $500 \mathrm{mg}$ PLGA was then added and dissolved completely with magnetic stirring. This solution was coated onto a GI stent using an electro-spinning machine (EBS ES-Biocoater; Nano NC, Seoul, South Korea), consisting of a high-voltage power supply, syringe pump, X-Y robotic system, and drum-roll collector. The silicone-membrane-covered metal stent was placed into the rolling collector, and polymer/drug solution in a syringe (NanoNC, 24G) was sprayed onto the siliconemembrane-covered metal stent (diameter $1 \mathrm{~cm}$, length $10 \mathrm{~cm}$, rolling speed $500 \mathrm{rpm}$, spray rate $100 \mu \mathrm{L} /$ minute, voltage $15 \mathrm{kV}$ ). Vorinostat-incorporated nanofiber membrane was coated onto the stent and then used as vorinostat nanofiber-coated stent. Any remaining solvent in the vorinostat nanofiber-coated stent was removed in a vacuum drying oven at room temperature for $24 \mathrm{~h}$. The final products were stored at $4^{\circ} \mathrm{C}$. For drug release studies and animal studies, vorinostat nanofiber membrane was carefully isolated from the stent and used.

Nanofiber membrane without vorinostat (empty nanofiber) was prepared using a similar method in the absence of vorinostat as described previously.

Drug contents and loading efficiency in the nanofibers were evaluated using an HPLC system as described previously. ${ }^{28}$ The Flexar HPLC system (Flexar HPLC system, PerkinElmer $^{\circledR}$, Waltham, MA, USA) was composed of a Solvent Manager 5-CH degasser, an autosampler, a quaternary LC pump, a column oven, and an UV/VIS detector. Chromatography was performed with a guard column (SecurityGuard ${ }^{\circledR}$ Guard Cartridge Kit; Phenomenex, Torrance, CA, USA) and a C18 column (Brownlee C18 ${ }^{\circledR}, 5 \mu \mathrm{m}, 150 \times 4.6$; PerkinElmer Inc., Waltham, MA, USA) at $37^{\circ} \mathrm{C}$. Vorinostat was isocratically eluted from $10 \mathrm{mg}$ vorinostat nanofibers using mobile phase (acetonitrile/0.1\% formic acid at a ratio of 22/78); vorinostat was detected at $241 \mathrm{~nm}$ (flow rate of mobile phase: $1 \mathrm{~mL} / \mathrm{min}$ ). Recorded chromatograms were integrated with the Chromera 2.1 system software. 
A

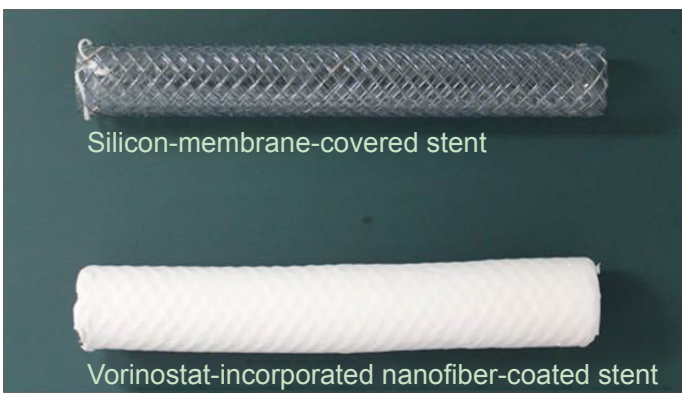

C

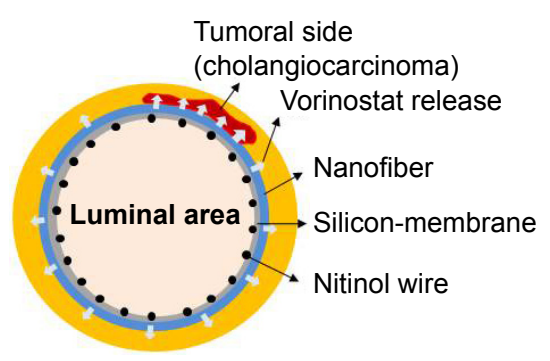

B
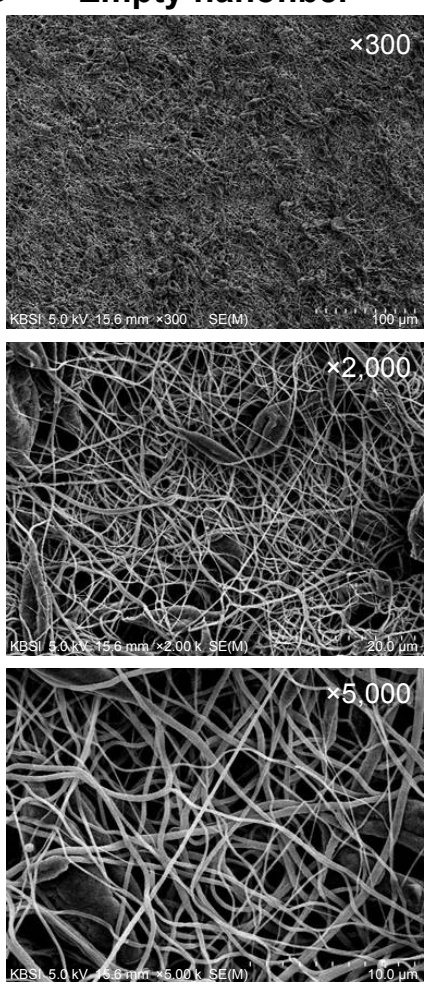

Vorinostat nanofiber
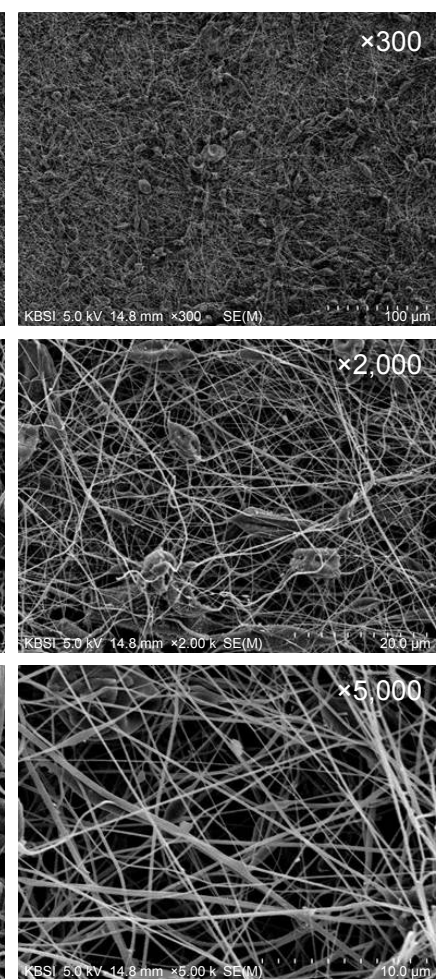

Figure I Vorinostat-eluting nanofiber-covered stent for CCA treatment.

Notes: (A) Vorinostat-incorporated nanofiber-coated Gl stent. (B) Morphological observation of empty nanofiber and vorinostat-incorporated nanofiber. (C) Schematic illustrations of cross-section of vorinostat-eluting nanofiber-covered stent in the bile duct region.

Abbreviations: CCA, cholangiocarcinoma; Gl, gastrointestinal.

Drug content and loading efficiency of vorinostat in the nanofibers were calculated as follows:

Drug content $=[($ Drug weight in the nanofiber $) /$ (Weight of nanofiber) $] \times 100$

Loading efficiency $=[($ Residual drug in the nanofiber)/(Initial feeding amount of drug) $] \times 100$

\section{Morphology}

Morphological observation of the nanofiber surface was examined with a field-emission scanning electron microscope (S-4800; Hitachi Co., Tokyo, Japan) at $25 \mathrm{kV}$.

\section{Drug release study}

Drug release was carried out using a phosphate-buffered saline (PBS, $10 \mathrm{mM}, \mathrm{pH} \mathrm{7.4)} \mathrm{solution} \mathrm{at} 37^{\circ} \mathrm{C}$. A $20 \mathrm{mg}$ nanofiber membrane was introduced into a falcon tube with $40 \mathrm{~mL}$ of PBS solution or RPMI 1640 media and placed in a shaking incubator (Multi Shaking Incubator, HB-201MS-4; Hanbaek Sci. Co., Bucheon-si, Korea) at 100 rpm. The released vorinostat was measured with an HPLC system as described previously. ${ }^{28}$ The equation of total released drug was as follows:

Total released drug $=[($ Weight of released drug $) /$ (Total weight of drug in the nanofiber membrane)] $\times 100$.

For comparison, similar weight of empty nanofiber membrane was used as a control, and its media were used to measure drug concentration as a blank test.

\section{Cell culture}

The HuCC-T1 CCA cell line was received from the Health Science Research Resources Bank (Osaka, Japan). SNU478, SNU245, and SNU 1196 CCA cell lines were obtained from Korean Cell Line Bank (Seoul, Korea). Cells were maintained in RPMI 1640 supplemented with $10 \%$ heat-inactivated FBS and $1 \%$ penicillin/streptomycin at $37^{\circ} \mathrm{C}$ in a $5 \% \mathrm{CO}_{2}$ incubator.

\section{Anticancer activity study}

CCA cells $\left(1 \times 10^{4}\right)$ were seeded in 96-well plates to anticancer activity. Cells were incubated in a $\mathrm{CO}_{2}$ incubator 
$\left(37^{\circ} \mathrm{C}\right)$ overnight. Following this, vorinostat in DMSO or vorinostat released from nanofibers was diluted with RPMI 1640 media and applied to cells. The anticancer activity study was carried out with serum-free RPMI 1640 media. For control treatment, cells were treated with $0.5 \%(\mathrm{v} / \mathrm{v})$ DMSO. For evaluation of anticancer activity of released vorinostat, vorinostat nanofibers $(20 \mathrm{mg})$ were immersed in serum-free RPMI 1640 media; vorinostat was eluted from nanofibers as described previously. One and 2 days later, supernatants of the media were diluted with serum-free RPMI 1640 media and added to cells. The concentration of eluted vorinostat was measured as described previously. Cell viability was assessed with the MTT proliferation assay. Twenty-four or $48 \mathrm{~h}$ later, $25 \mu \mathrm{L}$ MTT (5 mg/mL PBS, pH 7.4) solution was added to the cells, and the cells were further incubated for $4 \mathrm{~h}$. The medium was then removed, and $100 \mu \mathrm{L}$ DMSO was added to each well. This was gently shaken for 10 min. Cell viability was measured with an Infinite M200 Pro microplate reader (Tecan, Mannedorf, Switzerland) at $570 \mathrm{~nm}$. Viability was expressed as mean \pm standard deviation from eight wells.

\section{Apoptosis and necrosis analysis}

Cells seeded in 6 -well plates $\left(1 \times 10^{6}\right.$ cells per well) were treated with various concentrations of vorinostat released from vorinostat nanofibers and then incubated in a $\mathrm{CO}_{2}$ incubator overnight. For evaluation of drug stability in nanofibers, vorinostat was released from nanofibers using serumfree RPMI media for 1 day, 5 days, and 7 days. After that, vorinostat released from nanofibers was applied to cells for 1 day. The cells were harvested by trypsinization, washed with PBS, and suspended in $500 \mu \mathrm{L}$ of binding buffer. Cells were stained with FITC-conjugated Annexin V for the apoptosis assay and with propidium iodide (PI) for the necrosis analysis. Apoptosis and necrosis were analyzed by flow cytometry (BD Biosciences, San Jose, CA, USA).

\section{Western blotting}

Western blotting of CCA cells was performed as described previously. ${ }^{28}$ Cells treated for $24 \mathrm{~h}$ with vorinostat or vorinostat released from nanofibers were harvested by trypsinization, washed with cold PBS, and collected by centrifugation. Pellets were lysed in lysis buffer containing $50 \mathrm{mM}$ Tris, $150 \mathrm{mM}$ $\mathrm{NaCl}, 1 \%$ NP-40, 0.5\% deoxycholic acid, $0.1 \%$ sodium dodecyl sulfate (SDS) with phenylmethylsulfonyl fluoride, and a protease inhibitor cocktail (Roche Diagnostics $\mathrm{GmbH}$, Roche Applied Sci., Mannheim, Germany). This solution was centrifuged for $30 \mathrm{~min}$ at $4^{\circ} \mathrm{C}(14,000 \times g)$; the cell lysates (supernatant) were then used to measure protein concentration using the BCA Protein Assay kit (Pierce, Rockford, IL, USA). Protein $(50 \mu \mathrm{g})$ was loaded into SDS-polyacrylamide gel electrophoresis, transferred to a polyvinyl difluoride membrane, blocked with 5\% skim milk in TBS-T, probed with an appropriate primary antibody, and then treated with a secondary HRP-conjugated antibody for $1 \mathrm{~h}$. The immunoblots were detected by chemiluminescence and then quantified with digital analyses using the ImageJ software program.

\section{Antitumor activity of vorinostat- incorporated nanofiber in the animal tumor xenograft model}

Antitumor activity of vorinostat-incorporated nanofibercoated stent was assessed with HuCC-T1-bearing nude mice. HuCC-T1 cells $\left(1 \times 10^{7}\right)$ in $100 \mu \mathrm{L}$ of PBS were subcutaneously (s.c.) injected into the backs of nude mice ( 5 weeks old, male, 20-25 g in weight; Orient, Seongnam, South Korea). When the tumor size reached approximately $4 \mathrm{~mm}$ in diameter, an empty nanofiber and vorinostat-incorporated nanofiber were implanted under the solid tumor. The treatment dose was adjusted to $10 \mathrm{mg}$ vorinostat $/ \mathrm{kg}$. Mice were divided into four groups: control (untreated) group, PBS was s.c. injected beside tumor tissue; vorinostat injection group, vorinostat solution was s.c. injected beside tumor tissue (vorinostat dose: $10 \mathrm{mg} / \mathrm{kg}$ ); empty nanofiber group, an empty nanofiber was implanted under the solid tumor; vorinostat nanofiber group, a vorinostat-incorporated nanofiber was implanted under the solid tumor (vorinostat dose, $10 \mathrm{mg} / \mathrm{kg}$ ). For vorinostat injection, vorinostat was dissolved in ethanol/Cremophor $\mathrm{EL}^{\circledR}(1 / 1)$ mixed solution and then diluted ten times with PBS. For empty nanofiber and vorinostat nanofiber implantation, wafers of the same weight were cut and then implanted under the tumor tissue. For implantation of empty or vorinostat nanofibers, skin from the back of the mouse was excised $(0.5 \mathrm{~cm}$ in length). For comparison, skin from control treatment and vorinostat injected mice were also excised ( $0.5 \mathrm{~cm}$ in length). Each group consisted of four mice. Tumor volume was measured at 2 5-day intervals, and the first day of treatment was Day 0. Tumor volume was calculated with the following equation:

\section{$V=\left(a \times[b]^{2}\right) / 2 . a$ : largest diameter; $b$ : smallest diameter.}

All of the animal studies were conducted strictly according to the guidelines of the Pusan National University Institutional Animal Care and Use Committee (PNUIACUC). The animal protocol used in this study has been reviewed by 
the PNUIACUC in terms of ethical procedures and scientific care, and approved (approval number: PNU-2017-1608).

\section{Immunohistochemistry}

Solid tumors were isolated 26 days later and fixed in $4 \%$ formaldehyde. ${ }^{28}$ Tumor tissues were then paraffin-embedded and sliced for $\mathrm{H}$ and $\mathrm{E}$ staining. Immunohistochemical staining was carried out with acetyl histone $\mathrm{H} 3$ antibody at a dilution of 1:500, HDAC1 antibody at a dilution of 1:100, and HDAC3/HDAC4.5.7 antibodies at a dilution of 1:200. Staining was performed using an Envision kit (ThermoFisher Scientific, Waltham, MA, USA) according to the manufacturer's protocol.

\section{Statistical analysis}

Statistical analyses of the data from treated and untreated cells were performed using Student's $t$-test. A $p$-value $<0.05$ was considered to be statistically significant.

\section{Results}

\section{Characterization of vorinostat nanofiber- coated stent}

Vorinostat-incorporated nanofibers were coated onto a covered GI stent with an electro-spinning technique as shown in Figure 1. To adhere vorinostat-incorporated nanofibers onto the GI stent, a silicone-membrane-covered stent was used, and polymer/vorinostat solution was sprayed onto the covered GI stent (Figure 1A). As shown in Figure 1B, an empty nanofiber and vorinostat-incorporated nanofiber were fabricated successfully. Vorinostat nanofibers have more granules or particles in their structure compared to empty nanofibers, even though the fiber thickness of vorinostat nanofibers seem to be smaller than empty nanofibers (Figure 1B). Fiber diameters of both empty and vorinostat nanofibers were less than $1 \mu \mathrm{m}$. Figure $1 \mathrm{C}$ shows a schematic illustration of vorinostat release from vorinostat nanofibers in the bile duct region. Vorinostat nanofibers coated onto the silicone membrane-covered stent would have been in direct contact with tumor tissues. The silicone membrane on the metal stent blocked the loss of drug into the luminal space, allowing vorinostat to be efficiently delivered to the tumor tissue with minimal drug loss.

Drug contents and loading efficiency were $\sim 9.0 \%(\mathrm{w} / \mathrm{w})$ and $98.8 \%$, respectively. Drug contents between theoretical and experimental values were not significantly different, and most of the drug was successfully incorporated into nanofibers.

Drug release experiments were performed in PBS solution or cell culture media (RPMI 1640 media) as shown in Figure 2. Vorinostat nanofibers showed initial burst release for $8 \mathrm{~h}$ in cell culture media and $24 \mathrm{~h}$ in PBS solution. When cell culture media were adapted to the drug release study as shown in Figure 2A, the vorinostat release rate was very fast and almost all of the drug was liberated by 4 days. These results might be due to various nutrients in the cell culture media, such as salt, vitamin, or protein, that act as a surfactant and facilitated solubilization of vorinostat. The vorinostat release rate was relatively slow in PBS solution, and vorinostat was released over 10 days due to the hydrophobicity of vorinostat.

\section{Anticancer activity in vitro}

The vorinostat nanofiber-coated stent was assessed with CCA cells, including HuCC-T1, SNU478, SNU245, and SNU 1196. Vorinostat dissolved in DMSO or vorinostat
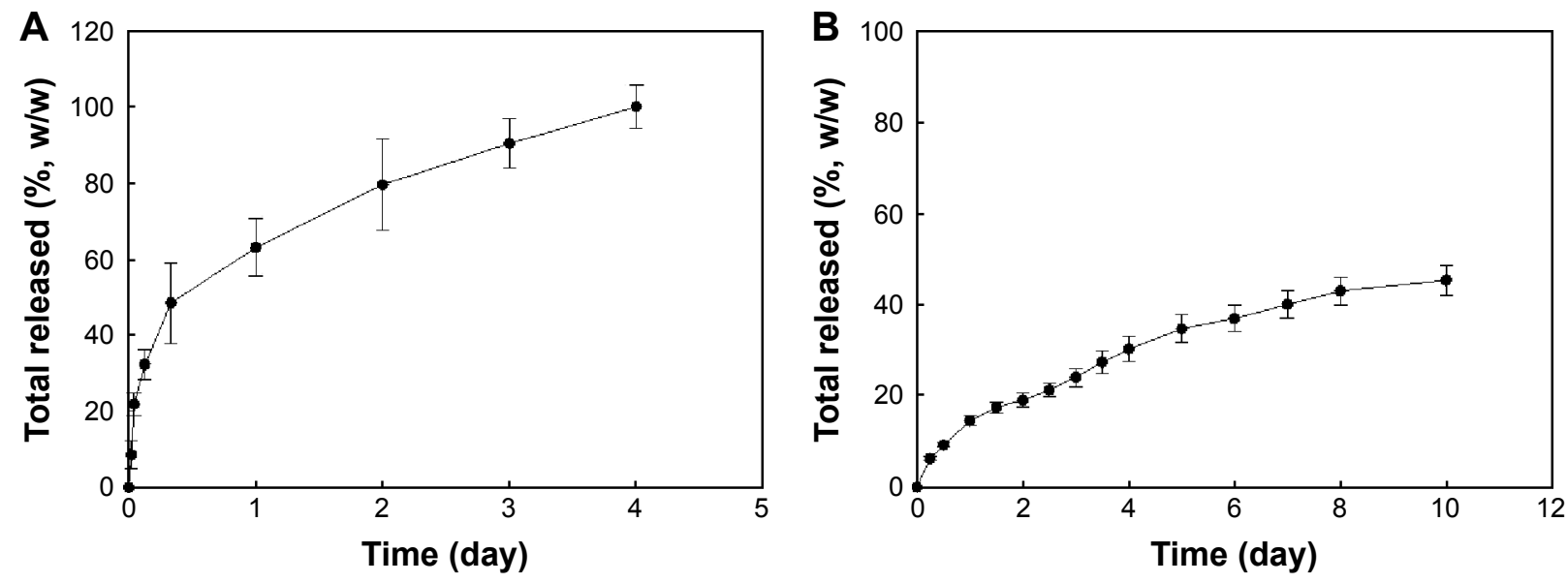

Figure 2 Vorinostat release from nanofiber membrane in cell culture media.

Notes: (A) RPMI 1640 media supplemented with 10\% FBS and antibiotics; (B) PBS solution.

Abbreviations: FBS, fetal bovine serum; PBS, phosphate-buffered saline. 

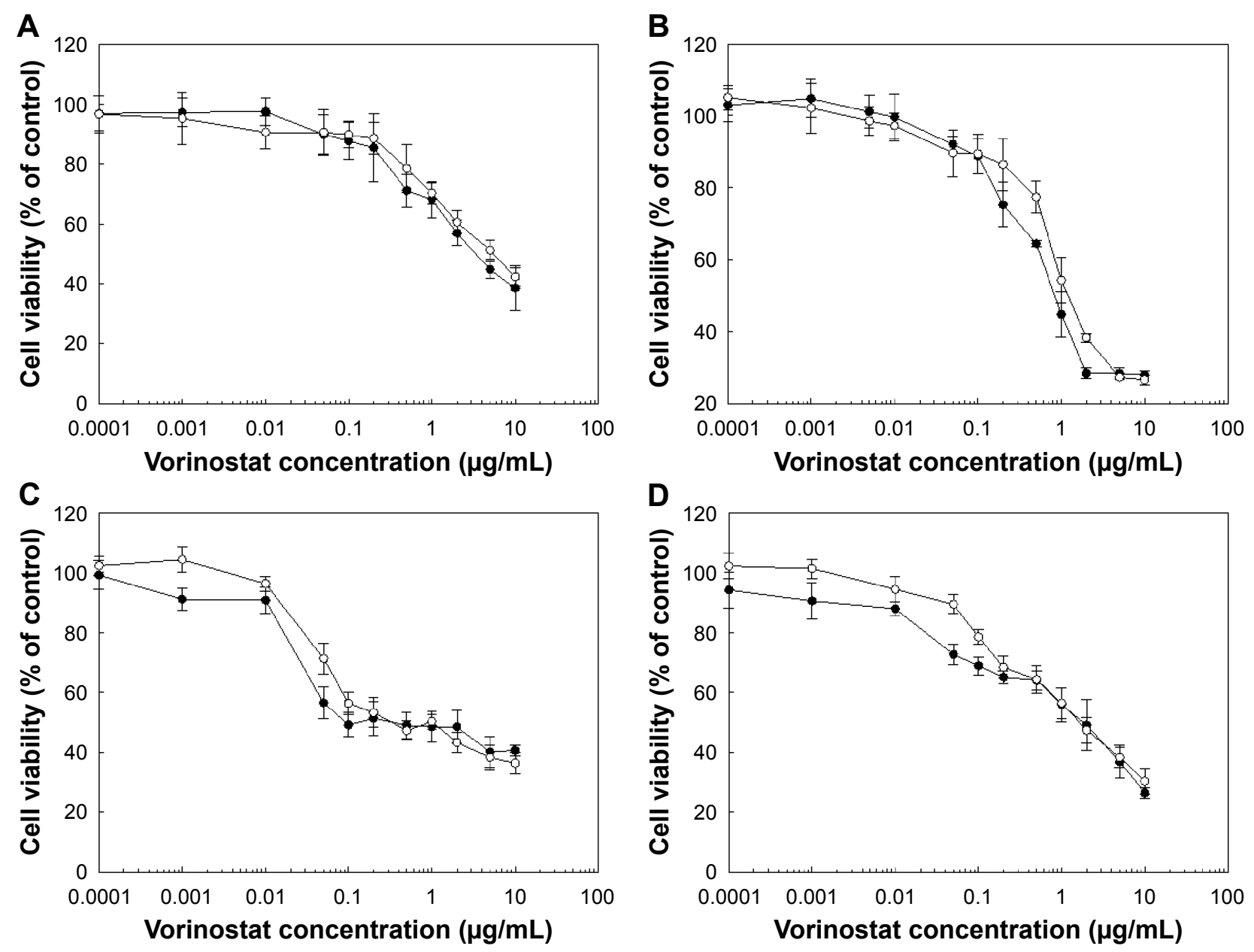

$\rightarrow$ Vorinostat $\multimap$ Released vorinostat

Figure 3 Anticancer activity of vorinostat and vorinostat released from nanofibers against various CCA cells.

Notes: (A) HuCC-TI, (B) SNU478, (C) SNU I 196, and (D) SNU245 CCA cells; I $10^{4}$ cells in 96-well plates were exposed to vorinostat or vorinostat released from nanofibers for 2 days.

Abbreviation: CCA, cholangiocarcinoma.

released from nanofibers was diluted with cell culture media and then added to cells. Cell viability is shown in Figures 3 and 4. As shown in Figure 3, CCA cell viability was dosedependently decreased according to vorinostat concentration. Vorinostat released from nanofibers showed similar anticancer efficacy to vorinostat itself, indicating that the intrinsic anticancer activity of vorinostat was not changed during the nanofiber fabrication process.

Figure 4 shows the effect of the vorinostat release period from nanofibers on the anticancer activity against HuCC-T1 and $\mathrm{SNu} 478$ cells in vitro. Vorinostat released from nanofibers at 1 day, 3 days, 5 days, and 7 days was used to assess anticancer activity and apoptosis/necrosis analysis against SNU478 and HuCC-T1 cells. As shown in Figure 4A, cell viability in vorinostat released from nanofibers was not significantly changed compared to vorinostat itself; the drug maintained anticancer activity after 7 days of the drug release experiment. Apoptosis/necrosis analysis also supported these results, as shown in Figure 4B. The number of apoptotic/necrotic cells was not significantly changed between Days 1, 5, and 7 of the drug release period compared to vorinostat itself. These results indicated that the intrinsic biological activity of vorinostat is maintained during the nanofiber fabrication process and drug release period.

Figure 5 shows the results of Western blotting of HuCC-T1 cells after treatment with vorinostat, empty nanofiber, and vorinostat nanofiber. To assess vorinostat activity in nanofibers, a vorinostat nanofiber was dissolved in DMSO and vorinostat released in media from nanofiber (Figure 2) was harvested. This solution was added to cells. As shown in Figure 5, expression of Ac-histone $\mathrm{H} 3$ and $\mathrm{H} 4$ was shown as a strong band when vorinostat was applied to HuCC-T1 


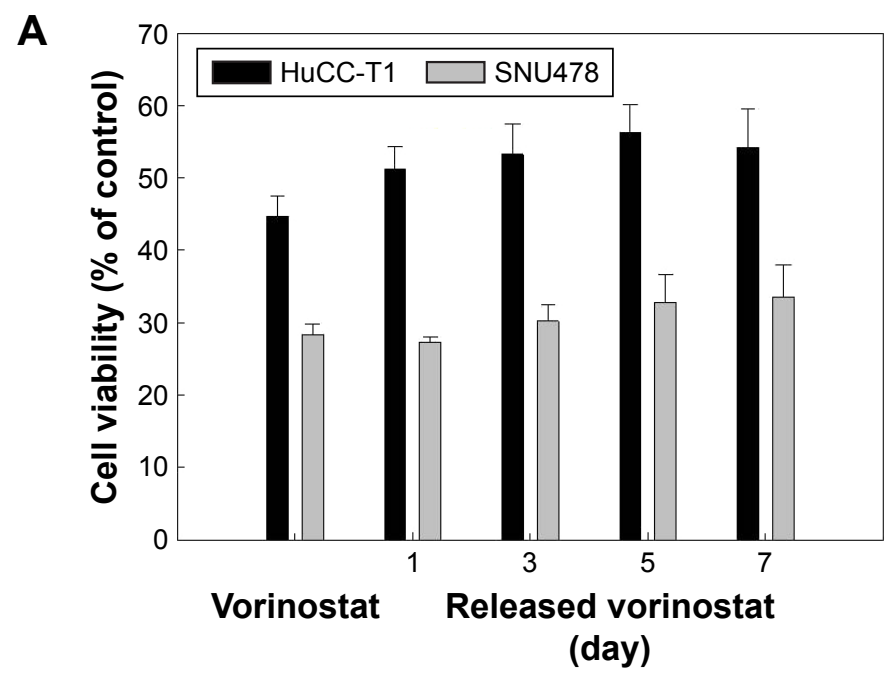

B
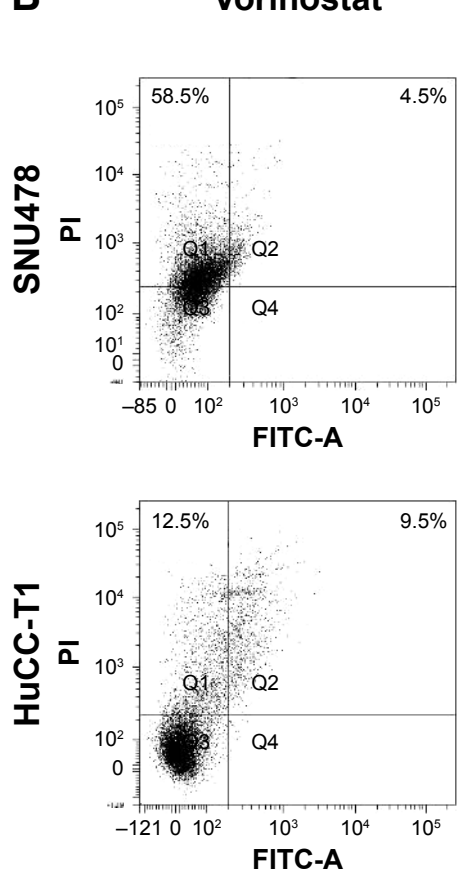

Released vorinostat
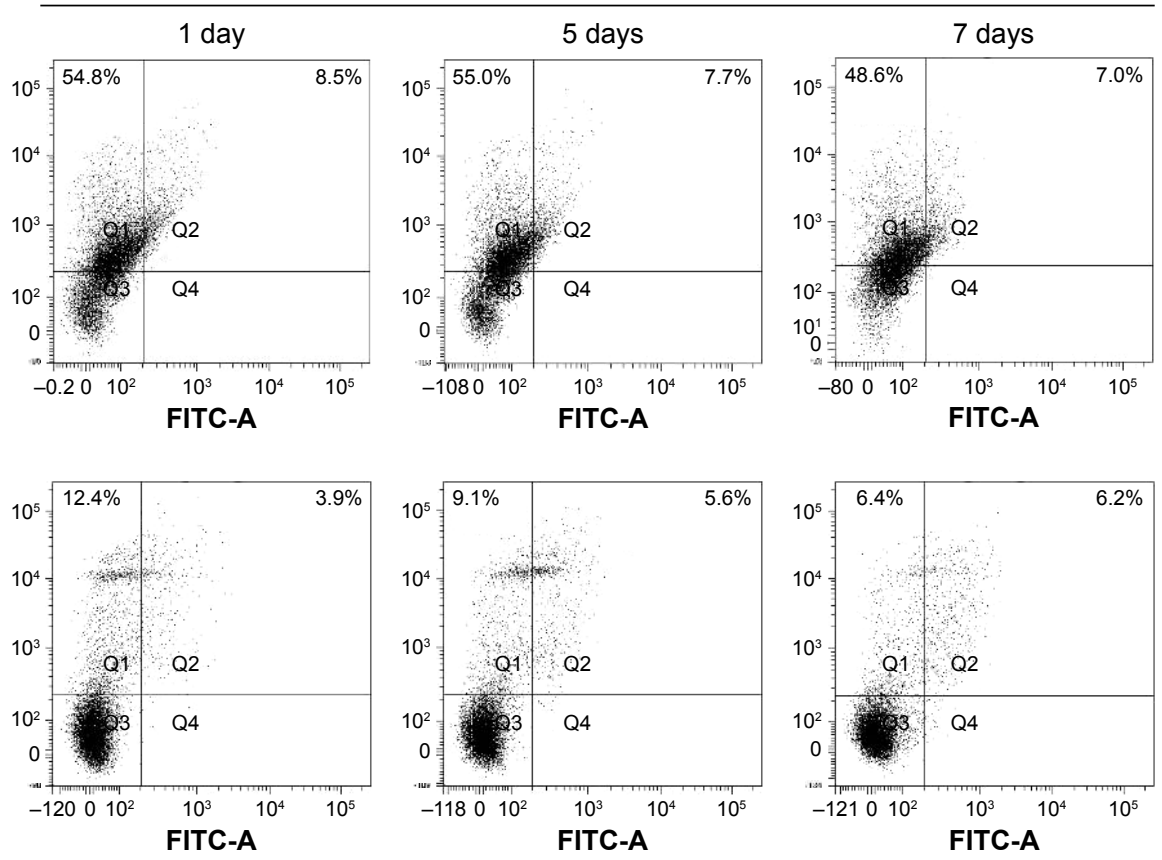

Figure 4 The effect of vorinostat and released vorinostat against HuCC-TI and SNU478 cells.

Notes: (A) Anticancer activity and (B) apoptosis/necrosis analysis. The supernatants were taken at I day, 3 days, 5 days, and 7 days of release experiment as described in experimental section. These solutions were used for released vorinostat treatment.

Abbreviation: PI, propidium iodide.

cells. Furthermore, HuCC-T1 cells showed decreased HDAC

$1,2,3$, and 4/5/7 expression after treatment of vorinostat. Both vorinostat dissolved in DMSO and vorinostat released in media also showed increased Ac-histone $\mathrm{H} 3 / \mathrm{Ha}$ and decreased HDAC 1.2.3.4/5/7 when added to cells. These results indicated that the intrinsic anticancer activity of vorinostat was maintained during the process of nanofiber fabrication and drug release study. Also, these results enabled us to predict anticancer activity of vorinostat nanofiber in in vivo study.

\section{Anticancer activity in vivo}

To assess anticancer activity of vorinostat nanofiber in vivo, a solid tumor xenograft was prepared by implantation of HuCC-T1 cells into the back of mice as shown in Figure 6. As shown in Figure 6, tumor volume of tumors treated with vorinostat nanofiber was noticeably smaller than those receiving control treatment, vorinostat injection or empty nanofiber, indicating that vorinostat in nanofiber was released continuously to tumor tissue and suppressed growth of solid tumors. Furthermore, expression of Ac-histone H4 was increased 


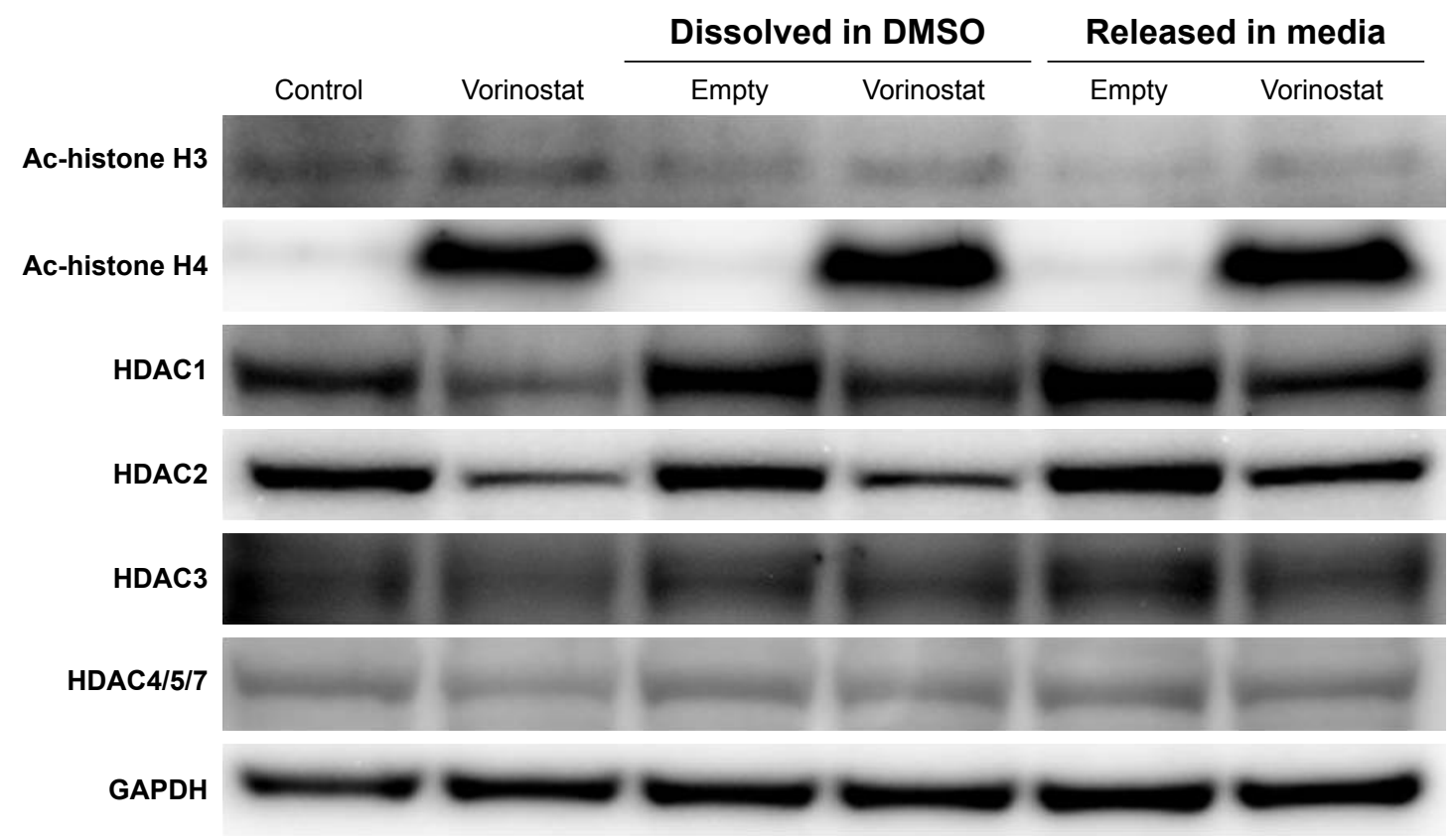

Figure 5 Western blot analysis of Ac-histone H3, H4, and HDACs' expression in HuCC-TI cells. Abbreviation: HDACs, histone deacetylases.

A

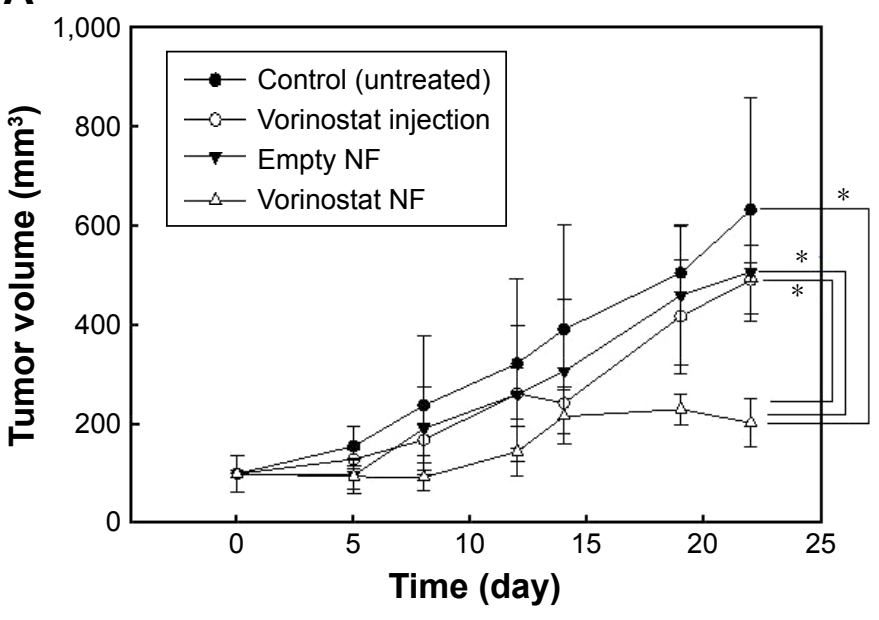

Control

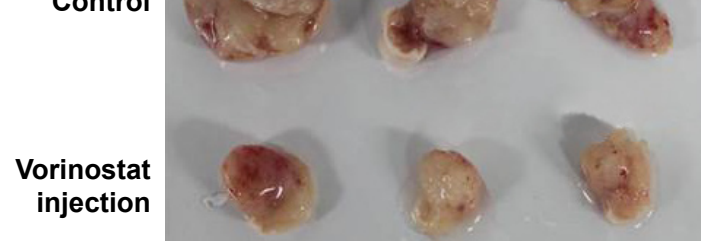

Vorinostat

NF

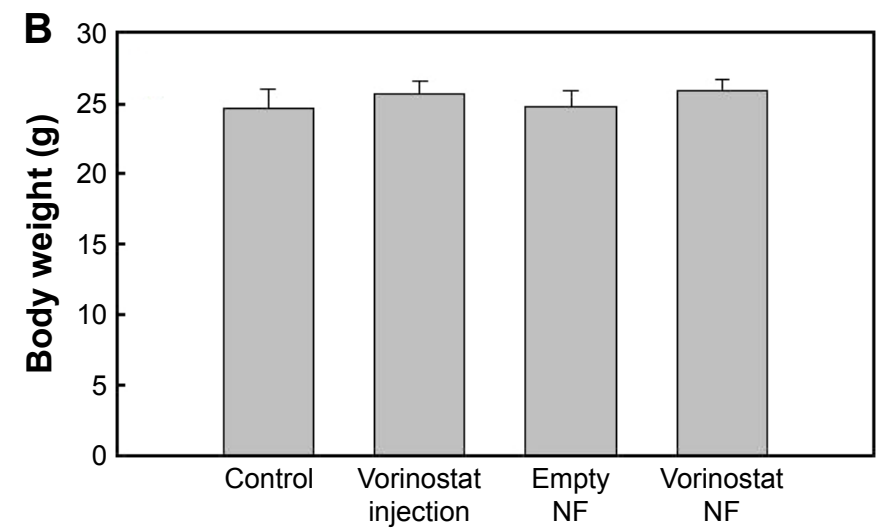

Figure 6 Antitumor activity of vorinostat-eluting nanofibers in HuCC-TI cell-bearing mice.

Notes: (A) Changes of tumor volume. (B) Body weight at 22 days. $* \mathrm{P}<0.05$. Vorinostat dose: $10 \mathrm{mg} / \mathrm{kg}$. PBS or vorinostat solution was s.c. injected beside tumor tissue for control (untreated) and vorinostat injection group, respectively. For empty nanofiber and vorinostat nanofiber implantation, wafers of the same weight were cut and then implanted under the tumor tissue.

Abbreviations: PBS, phosphate-buffered saline; s.c., subcutaneously; NF, nanofiber. 

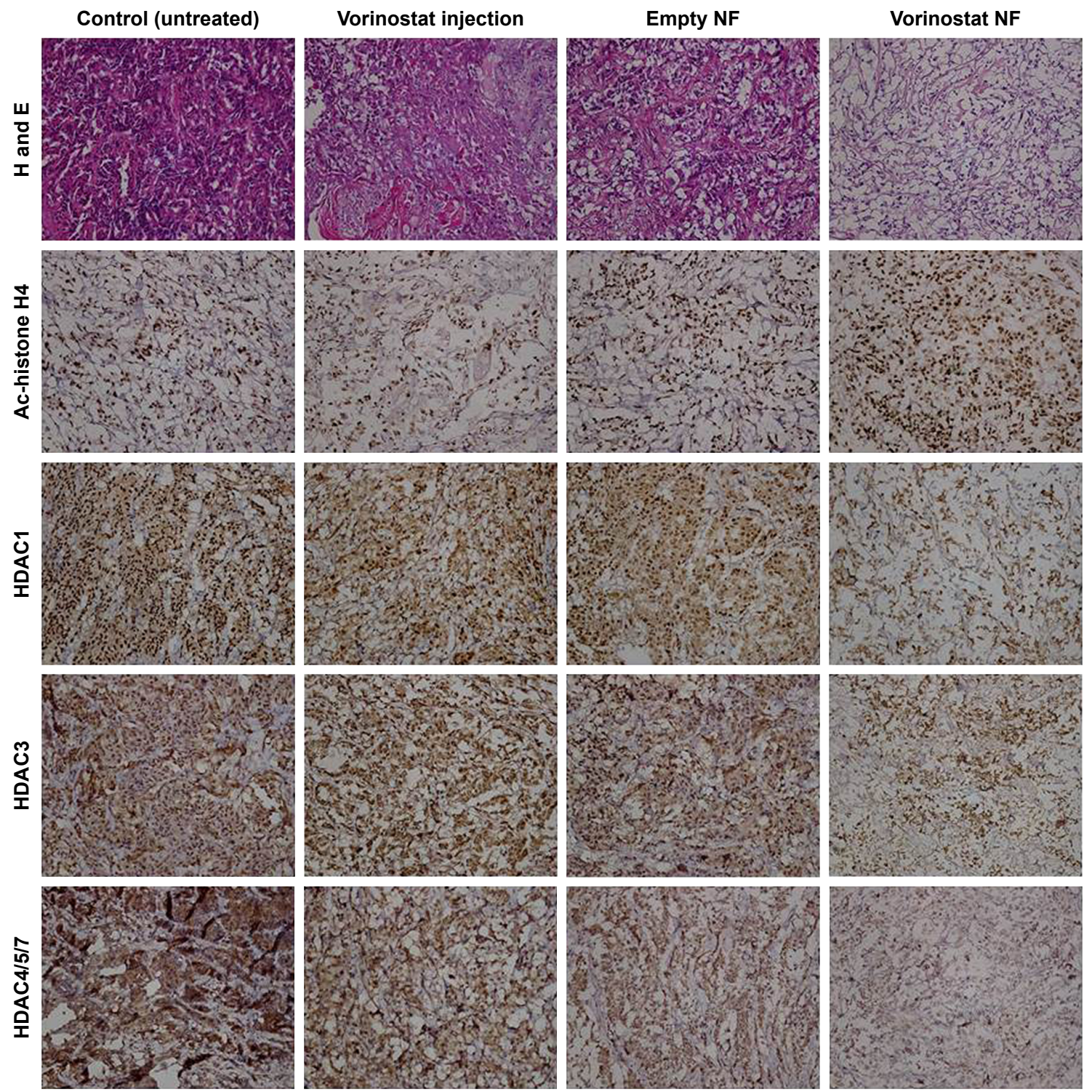

Figure 7 Immunohistochemistry $(\times 400)$ of HuCC-TI tumor tissues.

Note: To study HDAC expression, tumor tissues were stained with Ac-histone H4, HDACl, HDAC3, and HDAC4/5/7 antibodies.

Abbreviations: HDAC, histone deacetylase; NF, nanofiber.

with treatment of vorinostat nanofiber compared to control, vorinostat injection or empty nanofiber treatment, as shown in Figure 7. HDAC 1, 3, and 4/5/7 expression also decreased with treatment of vorinostat nanofiber, while control, vorinostat injection, and empty nanofiber showed increased HDAC expression. These results indicated that vorinostat nanofibers are effective in increasing acetylation of histone and suppressing HDAC expression. Therefore, vorinostat nanofiber inhibited CCA cells in vitro and in vivo.

\section{Discussion}

CCA is regarded as one of the most aggressive cancers, because patients with CCA are frequently diagnosed at an advanced stage with a median survival time of less than
24 months. ${ }^{29,30}$ Although surgical removal is generally considered as the best treatment option to prolong patient survival, less than one third of diagnosed CCA tumors can be surgically resected..$^{30}$ For unresectable CCA, conventional chemotherapy or radiotherapy can be considered as treatment option, ${ }^{30,31}$ however, these treatment options offer limited clinical benefit for the management of CCA patients. Placement of a metal stent for CCA patients is a palliative treatment option to maintain drainage of the bile duct and to prolong survival time. ${ }^{1,4}$ In terms of anticancer functionality, anticancer agent-eluting GI stents have been extensively investigated during the last decade. ${ }^{10-13,32-34}$ Paclitaxel-eluting stents for biliary obstruction have been developed for human application. ${ }^{32-34}$ Lee et al have reported safety issues related 
to anticancer agent-eluting stents used in a porcine model. ${ }^{32-35}$ They demonstrated the biological safety of DES and usefulness in local delivery of anticancer agent. Suk et al reported that the paclitaxel-eluting stent has feasibility, safety, and effectiveness as treatment for malignant biliary obstruction; ${ }^{33}$ however, the paclitaxel-eluting stent needs to be improved significantly in terms of anticancer efficacy and patency, because the paclitaxel-eluting stent showed negligible differences in stent patency and patient survivability compared to that of metal stents. ${ }^{33,34}$ We previously reported the anticancer efficacy of the sorafenib-eluting stent for CCA cells. ${ }^{13}$ Sorafenib effectively inhibited proliferation and migration of CCA cells in vitro and in vivo. Sorafenib was continuously released over 1 month, and anticancer activity was maintained for 30 days. In a tumor xenograft model using HuCC-T1 cells, the sorafenib-eluting stent effectively suppressed the tumor growth compared to control treatment.

Nanoscale materials such as nanoparticles, nanofibers, and polymeric conjugates are also highlighted in the application of DES. ${ }^{12,26,28,35}$ Among them, nanofiber mats are regarded as an ideal candidate for DES. ${ }^{12,26}$ Nanofibers have a unique microenvironment structure similar to that of a biological surface, easy to control drug release rate, and availability to incorporate various bioactive agents. ${ }^{36-38}$ Compared to other kinds of drug-eluting devices such as nanoparticles or microspheres, nanofiber mats provide a uniform coating layer on the GI stent; a simple coating process, ie, nanofiber mats can be coated onto GI stents by a one-step process using an electro-spinning machine, while nanoparticles or microspheres need to be separated from the dispersed solution with an additional process for stent coating. Furthermore, the malleable nature of nanofiber mats is essential for stent application, making them suitable devices for DES. ${ }^{39}$ Many investigators have evaluated nanofibers as a drug delivery device compared to nanoparticles. ${ }^{39-41}$ For example, Shan et al reported that nanofibers of methoxy poly(ethylene glycol)poly lactic acid copolymer provide better zero-order release kinetics of drugs, while burst drug release was observed from nanoparticles. ${ }^{40}$ For these reasons, drug-eluting nanofibers have received attention from many research groups for application in DES. ${ }^{12,26,36-38}$ Kim et al reported that a paclitaxel-eluting nanofiber-covered stent effectively inhibited colon cancer cells and extended stent patency. ${ }^{12}$ We also previously reported that a photosensitizer-eluting nanofiber-coated stent has efficacy against CCA cells. ${ }^{26}$ Vorinostat-incorporated nanofibers can be simply coated onto a covered stent (Figure 1) with an electro-spinning technique. This demonstrated the sustained release characteristics of vorinostat, as shown in Figure 2. Interestingly, vorinostat release behavior was significantly changed by media; most of the vorinostat was released over 4 days when RPMI 1640 cell culture media were used for drug release. The release rate of vorinostat was very slow, however, in PBS solution. The dominant mechanism of vorinostat release behavior might be diffusion of drugs into media; however, vorinostat release must be principally governed by a polymer degradation mechanism, because PLGA nanofibers with a 50:50 lactide:glycolide ratio is known to degrade linearly for up to 100 days. ${ }^{42}$ In that report, Dong et al also reported that the half-life of PLGA degradation was 80 days. ${ }^{42}$ In other reports, more than $30 \%$ of the molecular weight of PLGA (50:50, lactide:glycolide ratio) degraded for 30 days. ${ }^{43,44}$ Vorinostat release rate in PBS might be governed by degradation of polymers, except the period of initial burst of vorinostat.

Vorinostat nanofibers effectively inhibited the growth of HuCC-T1 tumors in a mouse xenograft model (Figure 6). Vorinostat released from nanofiber inhibited HDAC expression and induced acetylation of histone $\mathrm{H} 3 / \mathrm{H} 4$ in vitro and in vivo (Figures 5 and 7). We previously reported that vorinostat-incorporated nanoparticles have higher efficacy in suppression of tumor growth and induction of Ac-histone with suppression of HDAC protein compared to vorinostat itself, because vorinostat-incorporated nanoparticles remained in tumor tissue longer. ${ }^{28}$ In this study, vorinostat released from nanofibers was similar in acetylation of histone $\mathrm{H} 3 / \mathrm{H} 4$ and suppression of HDAC $1 \cdot 2 \cdot 3 \cdot 4 / 5 / 7$ compared to vorinostat itself (Figures 5 and 7). Since HDAC expression of CCA cells is closely related to advanced stage and poor prognosis, inhibition of its expression is a potential treatment candidate to prolong patient survivability. ${ }^{45}$ Furthermore, Boonjaraspinyo et al found that HDAC6 expression has a significant relationship with the stage of CCA. ${ }^{46}$ Gradilone et al also reported that HDAC6 inhibition induced a significant decrease in tumor growth. ${ }^{47}$ Although we did not show HDAC6 expression, vorinostat nanofibers showed positive results in inhibition of HDAC expression and induction of Ac-histone. These favorable results encourage future preclinical study and clinical feasibility.

\section{Conclusion}

In conclusion, we fabricated vorinostat nanofibers using biodegradable polymers with an electro-spinning technique. Vorinostat-incorporated nanofiber membrane was properly coated on the GI stent and formed nanofiber mats. Vorinostat was released from the nanofiber membrane over 1 week. Released vorinostat showed similar anticancer activity against 
various CCA cells in vitro compared to intact vorinostat. Like intact vorinostat, vorinostat released from nanofibers induced acetylation of histone $\mathrm{H} 3 / \mathrm{H} 4$ and inhibited HDAC $1 \cdot 2 \cdot 3 \cdot 4 / 5 / 7$ expression in vitro and in vivo. Furthermore, vorinostat nanofibers showed higher inhibition of tumor growth in HuCC-T1 tumor-bearing mice. We suggest that vorinostat nanofibers are a promising candidate for CCA treatment.

\section{Acknowledgment}

This study was supported by a grant from the Korean Health Technology R\&D Project, Ministry of Health \& Welfare, Republic of Korea (project no HI14C2220).

\section{Disclosure}

The authors report no conflicts of interest in this work.

\section{References}

1. Moole H, Bechtold ML, Cashman M, et al. Covered versus uncovered self-expandable metal stents for malignant biliary strictures: a metaanalysis and systematic review. Indian J Gastroenterol. 2016;35(5): 323-330.

2. Reim D, Friess H. Feeding challenges in patients with esophageal and gastroesophageal cancers. Gastrointest Tumors. 2016;2(4): 166-177.

3. Kim EJ, Kim YJ. Stents for colorectal obstruction: past, present, and future. World J Gastroenterol. 2016;22(2):842-852.

4. Li J, Li T, Sun P, et al. Covered versus uncovered self-expandable metal stents for managing malignant distal biliary obstruction: a meta-analysis. PLoS One. 2016;11(2):e0149066.

5. Ustundag Y, Bayraktar Y. Cholangiocarcinoma: a compact review of the literature. World J Gastroenterol. 2008;14(42):6458-6466.

6. Waseem D, Tushar P. Intrahepatic, perihilar and distal cholangiocarcinoma: management and outcomes. Ann Hepatol. 2017;16(1): 133-139.

7. Joo I, Lee JM. Imaging bile duct tumors: pathologic concepts, classification, and early tumor detection. Abdom Imaging. 2013;38(6): 1334-1350.

8. Freeman ML, Overby C. Selective MRCP and CT-targeted drainage of malignant hilar biliary obstruction with self-expanding metallic stents. Gastrointest Endosc. 2003;58(1):41-49.

9. Yasuda I, Mukai T, Moriwaki H. Unilateral versus bilateral endoscopic biliary stenting for malignant hilar biliary strictures. Dig Endosc. 2013;25 (Suppl 2):81-85.

10. Shatzel J, Kim J, Sampath K, et al. Drug eluting biliary stents to decrease stent failure rates: a review of the literature. World J Gastrointest Endosc. 2016;8(2):77-85.

11. Zhang Y, Ma L, Huang J, Shuang J, Chen J, Fan Z. The effect of paclitaxel-eluting covered metal stents versus covered metal stents in a rabbit esophageal squamous carcinoma model. PLoS One. 2017; 12(3): $\mathrm{e} 0173262$.

12. Kim SY, Kim M, Kim MK, et al. Paclitaxel-eluting nanofiber-covered self-expanding nonvascular stent for palliative chemotherapy of gastrointestinal cancer and its related stenosis. Biomed Microdevices. 2014;16(6):897-904.

13. Kim DH, Jeong YI, Chung CW, et al. Preclinical evaluation of sorafenibeluting stent for suppression of human cholangiocarcinoma cells. Int J Nanomedicine. 2013;8:1697-1711.

14. Richon VM. Cancer biology: mechanism of antitumour action of vorinostat (suberoylanilide hydroxamic acid), a novel histone deacetylase inhibitor. Br J Cancer. 2006;95 (Suppl 1):S2-S6.
15. Marks PA, Breslow R. Dimethyl sulfoxide to vorinostat: development of this histone deacetylase inhibitor as an anticancer drug. Nat Biotechnol. 2007;25(1):84-90.

16. Lee JH, Mahendran A, Yao Y, et al. Development of a histone deacetylase 6 inhibitor and its biological effects. Proc Natl Acad Sci U S A. 2013;110(39):15704-15709.

17. Rangwala S, Zhang C, Duvic M. HDAC inhibitors for the treatment of cutaneous T-cell lymphomas. Future Med Chem. 2012;4(4):471-486.

18. Doi T, Hamaguchi T, Shirao K, et al. Evaluation of safety, pharmacokinetics, and efficacy of vorinostat, a histone deacetylase inhibitor, in the treatment of gastrointestinal (GI) cancer in a phase I clinical trial. Int J Clin Oncol. 2013;18(1):87-95.

19. Sriraksa R, Limpaiboon T. Histone deacetylases and their inhibitors as potential therapeutic drugs for cholangiocarcinoma - cell line findings. Asian Pac J Cancer Prev. 2013;14(4):2503-2508.

20. Fakih MG, Pendyala L, Fetterly G, et al. A phase I, pharmacokinetic and pharmacodynamic study on vorinostat in combination with 5 -fluorouracil, leucovorin, and oxaliplatin in patients with refractory colorectal cancer. Clin Cancer Res. 2009;15(9):3189-3195.

21. Ree AH, Dueland S, Folkvord S, et al. Vorinostat, a histone deacetylase inhibitor, combined with pelvic palliative radiotherapy for gastrointestinal carcinoma: the Pelvic Radiation and Vorinostat (PRAVO) phase 1 study. Lancet Oncol. 2010;11(5):459-464.

22. He JC, Yao W, Wang JM, et al. TACC3 overexpression in cholangiocarcinoma correlates with poor prognosis and is a potential anti-cancer molecular drug target for HDAC inhibitors. Oncotarget. 2016;7(46): $75441-75456$.

23. Kim C, Jeong YI, Ngoc BT, et al. Synthesis and characterization of porous carbon nanofibers with hollow cores through the thermal treatment of electrospun copolymeric nanofiber webs. Small. 2007; 3(1):91-95.

24. Cho E, Kim C, Kook JK, et al. Fabrication of electrospun PVDF nanofiber membrane for Western blot with high sensitivity. Journal of Membrane Science. 2012;389(1):349-354.

25. Salehi S, Czugala M, Stafiej P, et al. Poly (glycerol sebacate)-poly ( $\varepsilon$-caprolactone) blend nanofibrous scaffold as intrinsic bio- and immunocompatible system for corneal repair. Acta Biomater. 2017;50: 370-380.

26. Yoo JJ, Kim C, Chung CW, Jeong YI, Kang DH. 5-aminolevulinic acid-incorporated poly(vinyl alcohol) nanofiber-coated metal stent for application in photodynamic therapy. Int J Nanomedicine. 2012;7: 1997-2005.

27. Zhou F, Jia X, Yang Y, et al. Nanofiber-mediated microRNA-126 delivery to vascular endothelial cells for blood vessel regeneration. Acta Biomater. 2016;43:303-313.

28. Kwak TW, Kim DH, Jeong YI, Kang DH. Antitumor activity of vorinostat-incorporated nanoparticles against human cholangiocarcinoma cells. J Nanobiotechnology. 2015;13:60.

29. Sandhu DS, Roberts LR. Diagnosis and management of cholangiocarcinoma. Curr Gastroenterol Rep. 2008;10(1):43-52.

30. Rahnemai-Azar AA, Weisbrod AB, Dillhoff M, Schmidt C, Pawlik TM. Intrahepatic cholangiocarcinoma: current management and emerging therapies. Expert Rev Gastroenterol Hepatol. 2017;11(5): 439-449.

31. Charbel H, Al-Kawas FH. Cholangiocarcinoma treatment. Curr Gastroenterol Rep. 2012;14(6):528-533.

32. Lee DK, Kim HS, Kim KS, et al. The effect on porcine bile duct of a metallic stent covered with a paclitaxel-incorporated membrane. Gastrointest Endosc. 2005;61(2):296-301.

33. Suk KT, Kim JW, Kim HS, et al. Human application of a metallic stent covered with a paclitaxel-incorporated membrane for malignant biliary obstruction: multicenter pilot study. Gastrointest Endosc. 2007;66(4): 798-803.

34. Jang SI, Kim JH, You JW, et al. Efficacy of a metallic stent covered with a paclitaxel-incorporated membrane versus a covered metal stent for malignant biliary obstruction: a prospective comparative study. Dig Dis Sci. 2013;58(3):865-871. 
35. Jang SI, Kim JH, Kim M, et al. Porcine feasibility and safety study of a new paclitaxel-eluting biliary stent with a Pluronic-containing membrane. Endoscopy. 2012;44(9):825-831.

36. Wang J, An Q, Li D, et al. Heparin and vascular endothelial growth factor loaded poly(L-lactide-co-caprolactone) nanofiber covered stent-graft for aneurysm treatment. J Biomed Nanotechnol. 2015;11(11):1947-1960.

37. Hwang CW, Johnston PV, Gerstenblith G, et al. Stem cell impregnated nanofiber stent sleeve for on-stent production and intravascular delivery of paracrine factors. Biomaterials. 2015;52:318-326.

38. Aguilar LE, Unnithan AR, Amarjargal A, et al. Electrospun polyurethane/Eudragit L100-55 composite mats for the $\mathrm{pH}$ dependent release of paclitaxel on duodenal stent cover application. Int J Pharm. 2015;478(1):1-8.

39. Goyal R, Macri LK, Kaplan HM, Kohn J. Nanoparticles and nanofibers for topical drug delivery. J Control Release. 2016;240:77-92.

40. Shan X, Liu C, Li F, Ouyang C, Gao Q, Zheng K. Nanoparticles vs. nanofibers: a comparison of two drug delivery systems on assessing drug release performance in vitro. Designed Monomers and Polymers. 2015;18(7):678-689.

41. Sridhar R, Lakshminarayanan R, Madhaiyan K, Amutha Barathi V, Lim KH, Ramakrishna S. Electrosprayed nanoparticles and electrospun nanofibers based on natural materials: applications in tissue regeneration, drug delivery and pharmaceuticals. Chem Soc Rev. 2015;44(3): $790-814$.
42. Dong Y, Yong T, Liao S, Chan CK, Stevens MM, Ramakrishna S. Distinctive degradation behaviors of electrospun polyglycolide, poly(DL-lactide-co-glycolide), and poly(L-lactide-coepsilon-caprolactone) nanofibers cultured with/without porcine smooth muscle cells. Tissue Eng Part A. 2010;16(1):283-298.

43. Keon KJ, Jeong YI, Jang MK, Lee CH, Nah JW. Norfloxacin release from surfactant-free nanoparticles of poly(DL-lactide-co-glycolide) and biodegradation. Polymer Korea. 2002;26:535-542.

44. Jeong YI, Shim YH, Song KC, Park YG, Ryu HW, Nah JW. Testosterone-encapsulated surfactant-free nanoparticles of poly(DLlactide-co-glycolide): Preparation and release behaviour. Bull Korean Chem Soc. 2002;23(11):1579-1584.

45. Morine Y, Shimada M, Iwahashi S, et al. Role of histone deacetylase expression in intrahepatic cholangiocarcinoma. Surgery. 2012;151(3): 412-419.

46. Boonjaraspinyo S, Boonmars T, Kaewkes S, et al. Down-regulated expression of HSP70 in correlation with clinicopathology of cholangiocarcinoma. Pathol Oncol Res. 2012;18(2):227-237.

47. Gradilone SA, Radtke BN, Bogert PS, Huang BQ, Gajdos GB, LaRusso NF. HDAC6 inhibition restores ciliary expression and decreases tumor growth. Cancer Res. 2013;73(7):2259-2270.
International Journal of Nanomedicine

\section{Publish your work in this journal}

The International Journal of Nanomedicine is an international, peerreviewed journal focusing on the application of nanotechnology in diagnostics, therapeutics, and drug delivery systems throughout the biomedical field. This journal is indexed on PubMed Central, MedLine, CAS, SciSearch ${ }^{\circledR}$, Current Contents ${ }^{\circledR} /$ Clinical Medicine,

\section{Dovepress}

Journal Citation Reports/Science Edition, EMBase, Scopus and the Elsevier Bibliographic databases. The manuscript management system is completely online and includes a very quick and fair peer-review system, which is all easy to use. Visit http://www.dovepress.com/ testimonials.php to read real quotes from published authors. 